\title{
Linear Viscoelasticity of Dumbbells Interacting via Gaussian Soft-Core Potential
}

\author{
Takashi UnEYAmA ${ }^{\dagger}$ \\ JST-PRESTO, and Center for Computational Science, Graduate School of Engineering, Nagoya University, \\ Furo-cho, Chikusa, Nagoya 464-8603, Japan \\ (Received: November 2, 2020)
}

\begin{abstract}
In polymer melts, the interaction between segments are considered to be screened and the ideal Gaussian chain statistics is recovered. The experimental fact that linear viscoelasticity of unentangled polymers can be well described by the Rouse model is naively considered as due to this screening effect. Although various theoretical models are based on the screening effect and the screening effect is believed to be reasonable, the screening effect cannot be fully justified on a solid theoretical basis. In this work, we study the screening effect by utilizing a simple dumbbell type model. We perform simulations for dumbbell systems in which particles interact via the Gaussian soft-core potential. We show that, if the density of dumbbells is high, the Gaussian soft-core interaction is actually screened and the static structures are well described by the ideal model without the Gaussian soft-core interaction. We also show that the relaxation moduli of interacting dumbbell systems approximately coincide to those of the non-interacting dumbbell systems. In the low density systems, we observe the deviations from the ideal non-interacting systems. For example, the relaxation moduli become relatively broad. However, the relaxation moduli of such systems can be decomposed into the relaxation modes by the Gaussian soft-core interaction and the bond. The bond relaxation mode can be successfully described by a single Maxwell relaxation with effective relaxation strength and time. Our results support a naive use of the Rouse model to analyze unentangled polymer melts.
\end{abstract}

Key Words: Linear viscoelasticity / Dumbbell model / Gaussian soft-core potential / Screening / Brownian dynamics

\section{INTRODUCTION}

Most of rheological properties of polymer melts can be attributed to the dynamics of polymer chains. A polymer melt consists of many polymer chains, and a polymer chain consists of monomers or segments. The segments in a polymer melt interact each other via the interaction potential such as the Lennard-Jones potential. Therefore, ultimately, the dynamics of a polymer melt can be described as the dynamics of strongly interacting particles. This description is formally correct but does not give us clear information on the dynamics and rheological properties of polymer melts. From this viewpoint, the dynamics of polymer chains will be too complex to theoretically analyze. To theoretically analyze the dynamics of polymer chains and compare some theoretical predictions with experimental data, we need to introduce some approximations (or simplifications).

If we consider just a single tagged polymer chain in a melt, and ignore the interactions between segments (except the bonds), we can construct theoretical models which are analytically tractable. The thus constructed models are often called single-chain type models or mean-field models. Naively, the

\footnotetext{
Corresponding author.

E-mail: uneyama@mp.pse.nagoya-u.ac.jp

Tel: $+81-52-788-6217$
}

ignorance of the interactions between segments is justified as follows. In a polymer melt, one tagged segment is surrounded by other segments. The segments are expected to be uniformly distributed around the tagged segment. Then the force acting on the tagged segment will be effectively canceled, and the segment will behave as if it feels no interaction potential. Such a screening picture is found in many textbooks, ${ }^{1-3)}$ and various properties of polymer melts are explained on the basis of the ideal chain statistics which is based on the screening picture. The screening picture can be experimentally justified by measuring several physical quantities for polymer melts. For example, the chain conformation in a polymer melt can be measured by scattering experiments and is known to be well described by the ideal chain model. This agreement is, however, theoretically not fully clear. The ignorance of the interaction between segments is not theoretically justified, but rather just assumed (at least currently).

The screening effect seems to work for dynamics. If we assume the dynamics of a single tagged chain in a polymer melt with the screening effect, the dynamics can be described by a single-chain model such as the Rouse model. ${ }^{1,4)}$ In the Rouse model, a tagged chain is modeled as a bead-spring chain and the dynamics of the bead is simply described by the overdamped Langevin equation. Various dynamical quanti- 
ties including the relaxation modulus can be straightforwardly calculated for the Rouse model. The relaxation modulus predicted by the Rouse model agrees well with the experimental relaxation modulus of an unentangled polymer melt. The Rouse type behavior is also observed in molecular dynamics simulations. ${ }^{5)}$ Thus, at least apparently, the screening picture seems to be reasonable.

However, the dynamics of strongly interacting particles is generally not that simple, and the applicability of the screening effect to the dynamics of polymer melts is not theoretically clear. Let us consider a single tagged chain in an unentangled polymer melt. This tagged polymer chain is interacting with surrounding polymer chains. When the tagged chain moves, it should push the surrounding chains to make vacancy. Then the dynamics of the tagged chain and the surrounding chains should be correlated, and we may observe the chain dynamics which is qualitatively different from the Rouse dynamics. The fact that the dynamics can be well described by the Rouse model implies that the dynamic correlation is effectively canceled in polymer melts. To the best of the author's knowledge, no theory successfully justifies this cancellation. The polymer chains in unentangled melts are rather strongly interacting, and it would be extremely difficult to study the dynamics analytically without approximations. Even in the case of simple liquids which have no intramolecular conformational degrees of freedom, the theoretical models become quite complicated. ${ }^{6)}$

In this work, we study some aspects of the screening effect by simulations for simple systems. Sometimes realistic polymer models are too detailed and complex to study physical mechanisms. Thus we limit ourselves to a simple model, although it may not be realistic. To express the relaxational dynamics of polymer chains, the dumbbell type model would be a suitable minimal model. Despite its simplicity, the dumbbell type model can describe various interesting dynamical behaviors of polymers ${ }^{7)}$ For example, by incorporating the modulation of the mobility or the friction coefficient under flow, various nonlinear rheological behavior can be reproduced analytically. ${ }^{8-10)}$ Even if we limit ourselves to the linear viscoelastic behavior, dumbbell models with fluctuating diffusivity (or the fluctuating friction coefficient) ${ }^{11,12)}$ or with the inertia effect ${ }^{13)}$ exhibit non-trivial interesting behavior. Therefore, we employ dumbbells inceracting via some potentials as a model system to study the screening effect. Since the dumbbell model is highly coarse-grained, the Lennard-Jones potential (or a similar potential with a core) is not suitable. Besides, we want to study how the strength of the interaction potential affects the screening effect. For our purpose, a soft coarse-grained interaction potential with a tunable interaction strength is required. In this work we employ the Gaussian softcore interaction potential. The Gaussian soft-core interaction allows polymers to fully overlap, and we can easily tune the interaction strength.

We perform simulations for systems with interacting dumbbells and study some static and dynamic properties including the linear viscoelasticity. We show that the screening of the interaction is actually observed in such a simple model. Especially, if the density of the system is sufficiently high, the static structure of a single tagged dumbbell in the system can be well described by the non-interacting, ideal dumbbell model without interaction. Also, the linear viscoelasticity of an interacting dumbbell system is well described by the corresponding ideal dumbbell system. We discuss how we can interpret the simulation results, and also discuss the validity of the naive screening picture for other coarse-grained polymer models.

\section{MODEL}

In this work, we employ the Brownian dynamics model originally developed for symmetric block copolymers. ${ }^{14)}$ We divide a single polymer chain into two subchains with the equal molecular weight. Then, we assume that a subchain is fully equilibrated and segments are distributed around the center of mass of the subchain. Then, the subchain would be interpreted as a soft particle with a Gaussian type kernel. ${ }^{15,16)}$ A polymer chain can be modeled as a dumbbell type molecule in which two soft particles are connected by a tethering potential.

We consider a system with the volume $V$ and number of polymer chains $M$. The number density of the particles is calculated as $\rho=2 M / V$. We express the positions of two soft particles in the $j$-th polymer as $\boldsymbol{R}_{j, 1}$ and $\boldsymbol{R}_{j, 2}$, respectively. The dimensionless units are convenient for simulations. We take the average size of the bond vectors for polymers, the thermal energy $k_{B} T$ ( $k_{B}$ and $T$ are the Boltzmann constant and the temperature), and the friction coefficient for a subchain, to be unity. In the dimensionless units, the interaction potentials can be expressed as:

$$
\begin{aligned}
& U\left[\left\{\boldsymbol{R}_{j, k}\right\}\right]=\sum_{j} \phi\left(\boldsymbol{R}_{j, 1}-\boldsymbol{R}_{j, 2}\right) \\
& +\sum_{(j, k)>(l, m)} v\left(\boldsymbol{R}_{j, k}-\boldsymbol{R}_{l, m}\right), \\
& \phi(\boldsymbol{r})=\frac{1}{2} \boldsymbol{r}^{2}, \\
& v(\boldsymbol{r})=\frac{\epsilon}{\pi^{3 / 2}} \exp \left(-\boldsymbol{r}^{2}\right) .
\end{aligned}
$$


In the second summation in eq (1), the expression $(j, k)>$ $(l, m)$ represents that the summation is taken only for $2 j+k>$ $2 l+m$. The potential functions $\phi(\boldsymbol{r})$ and $v(\boldsymbol{r})$ represent the tethering interaction and the Gaussian soft-core interaction, respectively. $\epsilon \geq 0$ represents the strength of the Gaussian soft-core interaction. We may call $\epsilon$ as the interaction strength in what follows. (In the case of $\epsilon=0$, there is no interaction between different polymer chains and thus the system reduces to the ideal dumbbell model. If the dumbbells do not interact each other, the system reduces to the canonical ensemble of harmonic dumbbells.)

As the dynamics model, we employ a simple overdamped Langevin equation. In absence of the external flow field, the dynamic equation is given as the following Langevin equation (in dimensionless units):

$$
\frac{d \boldsymbol{R}_{j, k}(t)}{d t}=-\frac{\partial U\left(\left\{\boldsymbol{R}_{j, k}\right\}\right)}{\partial \boldsymbol{R}_{j, k}}+\sqrt{2} \boldsymbol{w}_{j, k}(t) .
$$

Here, $\boldsymbol{w}_{j, k}(t)$ is the Gaussian white noise which satisfies the fluctuation-dissipation relation. The first and second order moments of $\boldsymbol{w}_{j, k}(t)$ become

$$
\left\langle\boldsymbol{w}_{j, k}(t)\right\rangle=0, \quad\left\langle\boldsymbol{w}_{j, k}(t) \boldsymbol{w}_{l, m}(t)\right\rangle=\delta_{j l} \delta_{k m} \mathbf{1} \delta\left(t-t^{\prime}\right)
$$

where $\langle\ldots\rangle$ represents the statistical average and $\mathbf{1}$ is the unit tensor. The numerical simulations are performed by discretizing the Langevin equation (4) and numerically integrate it. We employ the second order stochastic Runge-Kutta scheme ${ }^{17)}$ to accurately integrate the Langevin equation. The details of the numerical scheme can be found in the previous work. ${ }^{14)}$

The stress tensor of the system can be calculated as

$$
\begin{aligned}
& \hat{\boldsymbol{\sigma}}=\frac{1}{V} \sum_{j, k, l, m} \frac{\partial U\left(\left\{\boldsymbol{R}_{j, k}\right\}\right)}{\partial \boldsymbol{R}_{j, k}} \boldsymbol{R}_{j, k}-\rho \mathbf{1} \\
&=\frac{1}{V} \sum_{j}\left(\boldsymbol{R}_{j, 1}-\boldsymbol{R}_{j, 2}\right)\left(\boldsymbol{R}_{j, 1}-\boldsymbol{R}_{j, 2}\right) \\
& \quad+\frac{\epsilon}{V \pi^{3 / 2}} \sum_{(j, k)>(l, m)} \exp \left[-\left(\boldsymbol{R}_{j, k}-\boldsymbol{R}_{l, m}\right)^{2}\right] \\
& \quad \times\left(\boldsymbol{R}_{j, k}-\boldsymbol{R}_{l, m}\right)\left(\boldsymbol{R}_{j, k}-\boldsymbol{R}_{l, m}\right)-\rho \mathbf{1} .
\end{aligned}
$$

If we ignore the contribution of the Gaussian soft-core interaction, the stress tensor reduces to the widely utilized form which is consistent with the stress-optical rule. In this work, we want to explore how the Gaussian soft-core interaction affects the linear viscoelasticity, and thus we utilize eq (6) without any further approximations.

In equilibrium, the stress tensor becomes isotropic on average. This can be related to the pressure of the system, $P$, as

$$
\langle\hat{\boldsymbol{\sigma}}\rangle_{\mathrm{eq}}=-P \mathbf{1},
$$

where $\langle\ldots\rangle_{\text {eq }}$ represents the statistical average over the equilibrium distribution. If the Gaussian soft-core interaction is absence, the pressure can be exactly calculated because the system behaves as an ideal gas. But for the interacting dumbbell systems, we should introduce some approximations to obtain the analytic expression.

The linear viscoelasticity of this model can be numerically calculated by the Green-Kubo formula. The stress tensor is a function of the particle positions and thus it can be interpreted as a function of time. The Green-Kubo formula states that the shear relaxation modulus is given as the correlation function of the stress tensor: ${ }^{18)}$

$$
G(t)=V\left\langle\hat{\sigma}_{x y}(t) \hat{\sigma}_{x y}(0)\right\rangle_{\mathrm{eq}} .
$$

We numerically calculate the relaxation modulus by using eq (8) with eq (6).

In order to estimate the contribution of the soft-core interaction to various properties of an interacting dumbbell system, we consider a system without the tethering potential as a reference system. We may call this reference model as the soft particle model. The soft particle system consists of $M$ particles, and the $j$-th particle position is expressed as $\boldsymbol{R}_{j}$. The particle density of the system is $\rho=M / V$. The interaction energy of the soft particle model is given as

$$
U\left[\left\{\boldsymbol{R}_{j}\right\}\right]=\sum_{j>k} v\left(\boldsymbol{R}_{j}-\boldsymbol{R}_{k}\right)
$$

instead of eq (1). Also, the stress tensor of the soft particle model is given as

$$
\begin{array}{r}
\hat{\boldsymbol{\sigma}}=\frac{\epsilon}{V \pi^{3 / 2}} \sum_{j>k} \exp \left[-\left(\boldsymbol{R}_{j}-\boldsymbol{R}_{k}\right)^{2}\right] \\
\times\left(\boldsymbol{R}_{j}-\boldsymbol{R}_{k}\right)\left(\boldsymbol{R}_{j}-\boldsymbol{R}_{k}\right)-\rho \mathbf{1} .
\end{array}
$$

instead of eq (6). Dynamic equation (4) is essentially the same (only the subscript $j k$ is changed to $j$ ).

We perform simulations with several systems with different particle densities and the interaction strengths. We fix the system size to be $8^{3}$ and apply the periodic boundary condition to all the directions. We change the number of polymers $M$ and the interaction parameter $\epsilon$. We vary $M$ so that the particle density $\rho$ varies as $\rho=0.25,0.5,1,2$, and 4 . We also vary $\epsilon$ as $\epsilon=1,2,4,8,16,32$, and 64. For comparison, 
we perform simulations for non-interacting systems by simply setting $\epsilon=0$. Also, for comparison, we perform simulations for soft particle systems which have the same parameters as the interacting dumbbell systems. The time step size is set to be $\Delta t=0.01$, and simulations are performed for sufficiently long time. (The stochastic Runge-Kutta scheme is a weak second order scheme, and this time step is sufficiently small in most cases.) Simulations are conducted with the house-made code which is available at the author's web site. ${ }^{19)}$ To improve the statistical accuracy, the simulations for the same $\rho$ and $\epsilon$ with different random number seeds are performed and the averages over different seeds and time are taken. For the calculation of the relaxation modulus, we employ Likhtman's formula to further improve the statistical accuracy. ${ }^{20)}$

\section{RESULTS}

\subsection{Static Properties}

Before we study the linear viscoelasticity, we study some static properties. The static structure of the system can be characterized by the radial distribution function (RDF):

$$
g(r)=\frac{1}{4 \pi \rho r^{2}} \sum_{(j, k)>(l, m)}\left\langle\delta\left(r-\left|\boldsymbol{R}_{j, k}-\boldsymbol{R}_{l, m}\right|\right)\right\rangle_{\mathrm{eq}} .
$$

In a non-interacting ideal dumbbell system, we have $g_{\text {id }}(r)=$ $1+(1 / \rho) \exp \left(-r^{2} / 2\right)$. The second term comes from the tethering potential. (In a non-interacting soft particle system, we simply have $g_{\mathrm{id}}(r)=1$.) A particle is connected to its partner by the tethering potential, and should be distributed near $r=0$. The contribution of the tethering potential becomes negligibly small if the density is sufficiently high: $g_{\text {id }}(r) \approx 1$. The RDF of a system is calculated as a normalized histogram. Notice that the RDF near $r=0$ is not accurate, because the number of samples becomes small for small $r$. (This is not serious in most of molecular dynamics simulations, because the most of interaction potentials have hard-core and the RDF becomes zero near $r=0$. In our model, however, the softcore interaction allows particles to overlap and the RDF can be non-zero even at $r=0$.) Figures 1 and 2 show the RDFs for interacting dumbbell systems and soft particle systems, with various densities and interaction parameters. The RDFs for the interacting dumbbell and soft particle systems with the same density and interaction parameter are quite similar. For the interacting dumbbell systems with the low density and low interaction parameter (Fig. 1(a)), we observe the increase of the RDF near $r=0$. This is the contribution of the tethering potential. Except this contribution of the tethering interaction, the RDF of an interacting dumbbell system is almost the
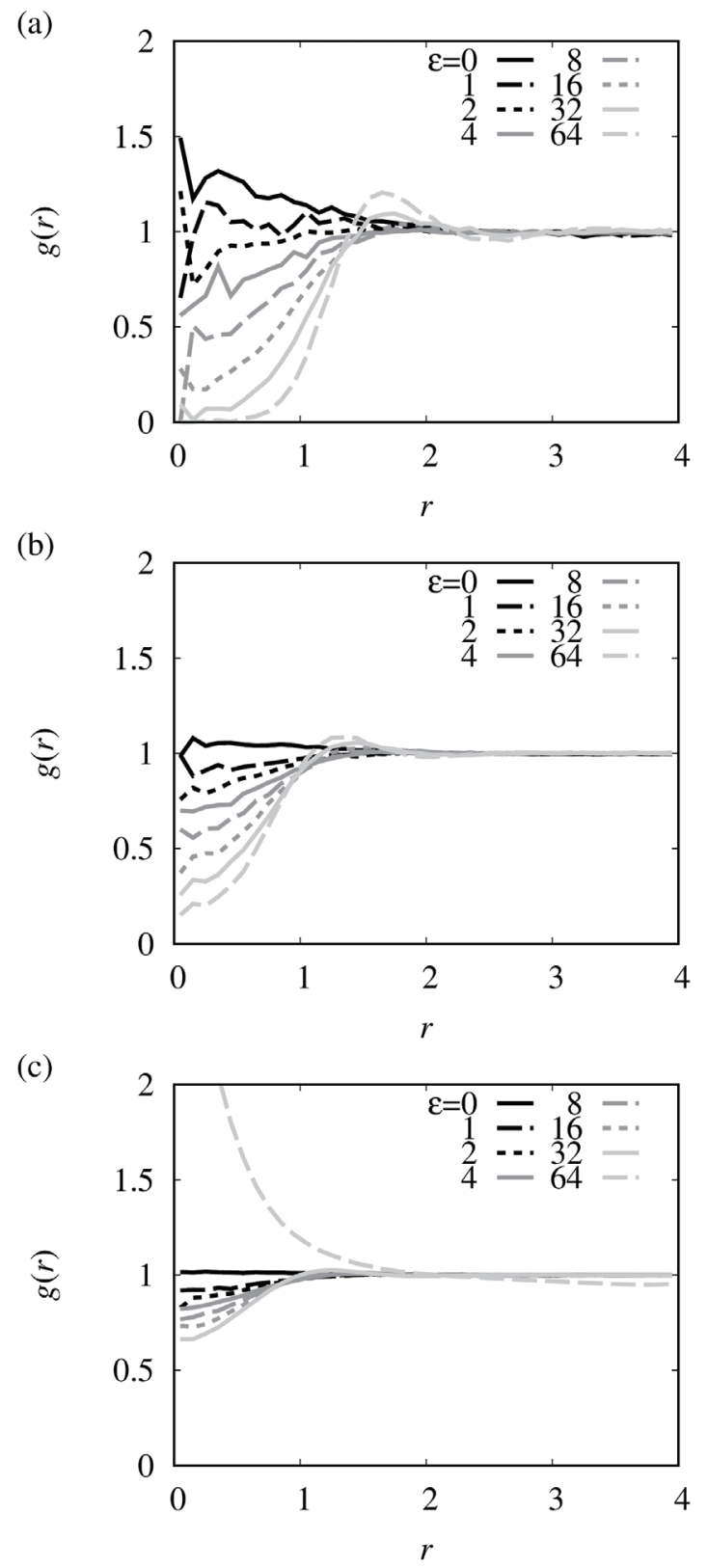

Fig. 1 The radial distribution functions for the interacting dumbbell systems. (a) $\rho=0.25$, (b) $\rho=1$, and (c) $\rho=4$. The data near $r=0$ are scattered due to small number of statistical samples.

same as that of the corresponding soft particle system. Thus we find that the static structure is essentially controlled by the Gaussian soft-core interaction in these systems.

For the system with the highest density and the highest interaction parameter ( $\rho=4$ and $\epsilon=64$ ) among the examined parameter sets, we observe that the RDF behaves in a qualitatively different way from other cases. The RDF increases largely as $r$ approaches to zero. This would be interpreted as the formation of dimer-like clusters. ${ }^{21,22)}$ The formation of clusters implies that the interaction is too strong in this system. We cannot exclude the possiblity that the simulation model and numerical scheme employed in this work do not work correctly and have produced artificial cluster structures. 
Anyway, we consider the case with $\rho=4$ and $\epsilon=64$ as an exceptional case, and do not directly compare this case with other cases in what follows. Except this special case, we observe that the RDF changes systematically as the density and the interaction parameter changes. The dependence of the RDF on the interaction parameter becomes weaker as the density increases. Thus we expect that, at the high density limit, the $\mathrm{RDF}$ will reduce to the ideal form $g(r) \approx g_{\text {id }}(r)$ independent of the interaction parameter.

To study static statistical properties of dumbbell conformations, we calculate the bond length distribution function. We define the bond length distribution function as
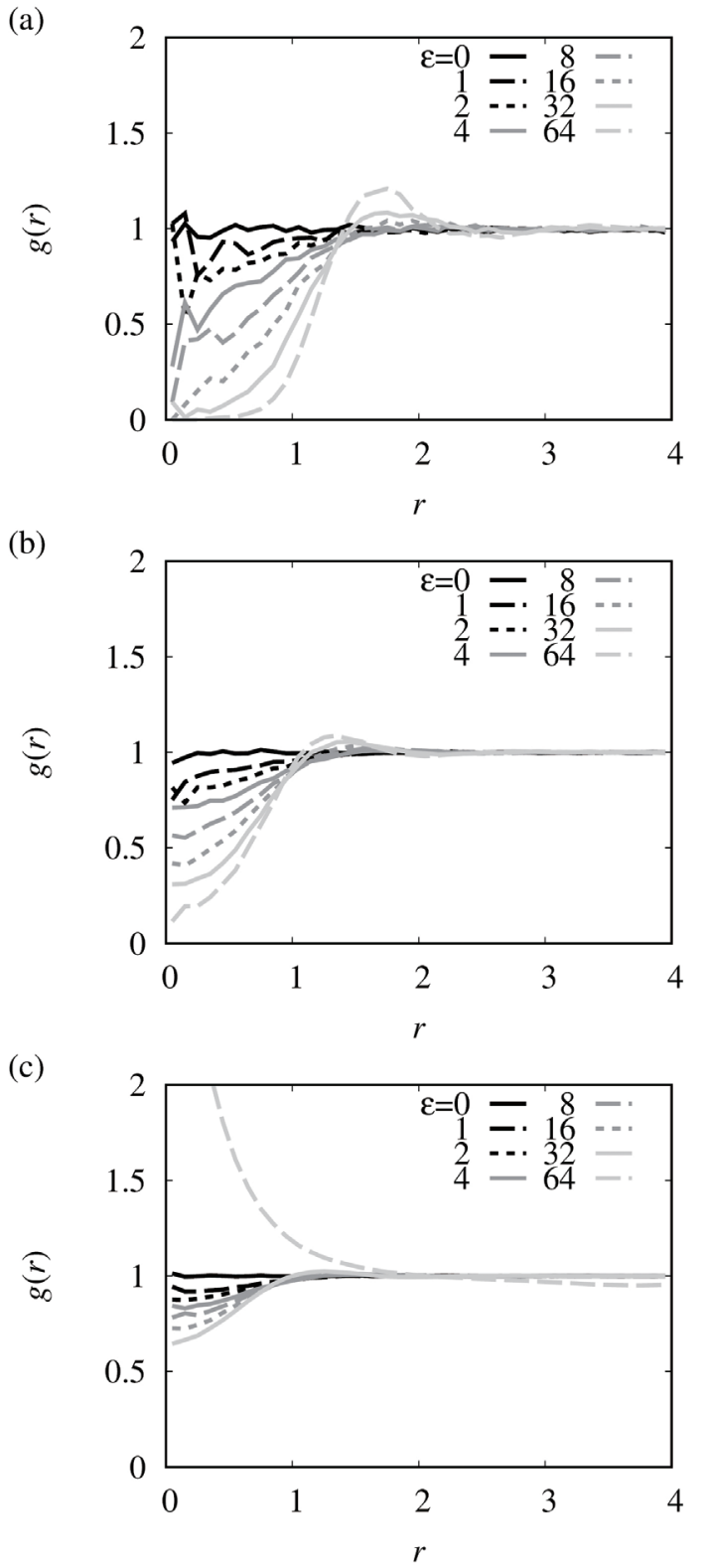

Fig. 2 The radial distribution functions for the soft particle systems. (a) $\rho=0.25$, (b) $\rho=1$, and (c) $\rho=4$. Simulation parameters are the same as the interacting dumbbell systems shown in Fig. 1.

$$
\Psi(Q)=\frac{1}{N} \sum_{j}\left\langle\delta\left(Q-\left|\boldsymbol{R}_{j, 1}-\boldsymbol{R}_{j, 2}\right|\right)\right\rangle_{\mathrm{eq}}
$$

In absence of the Gaussian soft-core interaction potential, we simply have $\Psi_{\text {id }}(Q)=\sqrt{2 / \pi} Q^{2} \exp \left(-Q^{2} / 2\right)$. If the equilibrium conformation is modulated by the Gaussian soft-core interaction, the bond length distribution should deviate from the equilibrium distribution. Figure 3 shows the bond length distribution functions for the interacting dumbbell systems. (Note that the curves for $\epsilon=0$ corresponds to the ideal distribution function $\Psi_{\mathrm{id}}(Q)$.) If the density is low, we observe that
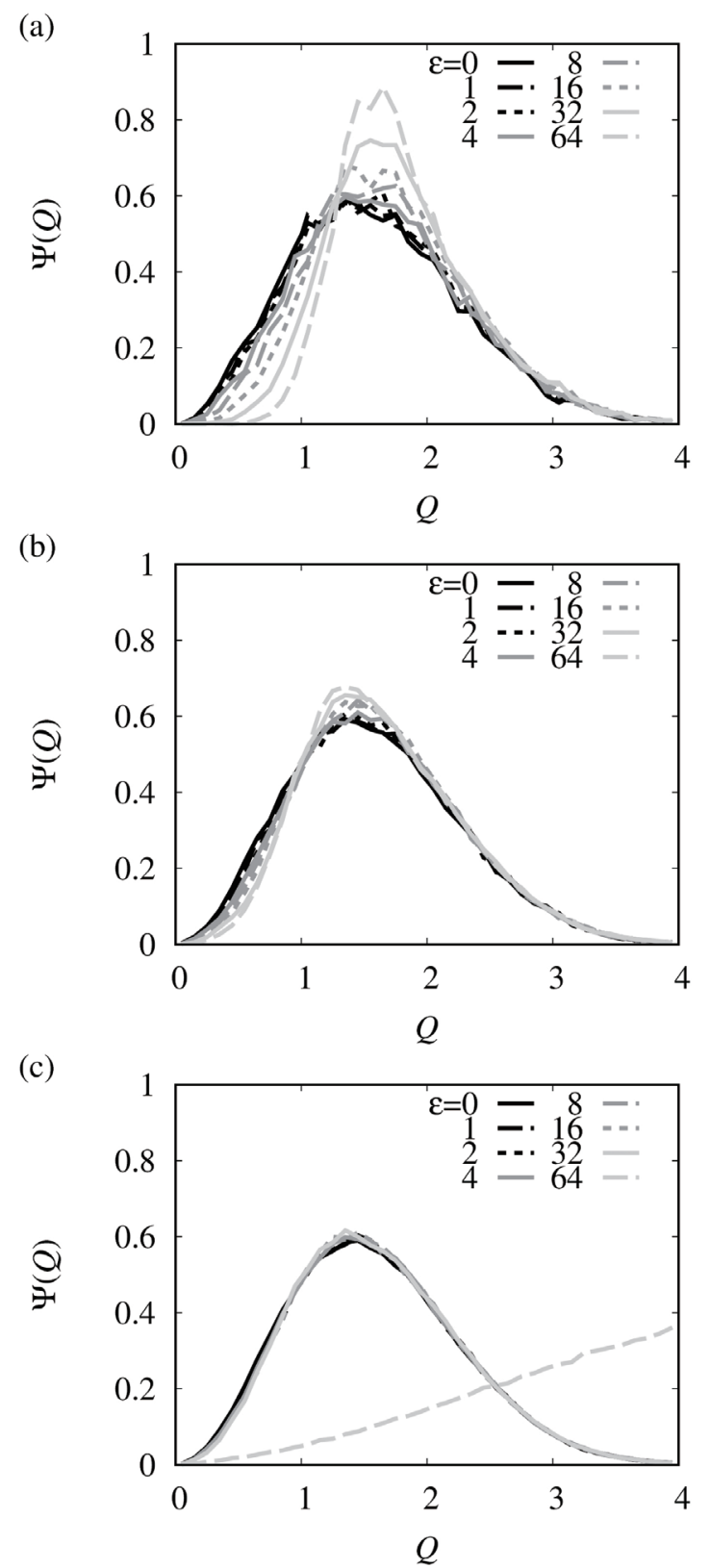

Fig. 3 The bond length distribution functions for the interacting dumbbell systems. (a) $\rho=0.25$, (b) $\rho=1$, and (c) $\rho=4$. Curves for $\epsilon=0$ (solid black curves) correspond to the distribution function for the ideal system $\Psi_{\text {id }}(Q)$. 
the bond length distribution clearly depends on the interaction parameter. As the density increases, the dependence of the bond length distribution on the interaction parameter becomes weak. The distribution for the exceptional case $(\rho=4$ and $\epsilon=64)$ is much different from other distributions as the case of the RDF. (Judging from this bond length distribution, the systems size $8^{3}$ seems to be too small in this case. Additional simulations for larger systems should be carefully performed, if we analyze the data for $\rho=4$ and $\epsilon=64$.) Except this case, we observe that the bond length distribution is almost independent of $\epsilon$ for the high density systems $(\rho=4)$, and at the high density limit we will have $\Psi(Q) \approx \Psi_{\text {id }}(Q)$. These results are consistent with the RDF data.

For low density systems, both the RDF and the bond length distribution function clearly depends on the interaction parameter. Such a behavior is widely observed for systems interacting via such as the Lennard-Jones potential. Our results show that as the density increases, the distributions become less sensitive to the interaction parameter. The equilibrium conformational statistics of a single tagged dumbbell can be well described by that for the corresponding non-interacting dumbbell. We may interpret this as the screening effect, because the RDF will approach to that of the non-interacting ideal system if the interaction between particles are effectively canceled. From the RDF and bond length distribution data, therefore we conclude that the screening effect actually works in our model.

The Gaussian soft-core interaction between dumbbells would directly affect some macroscopic thermodynamic quantities. Here we examine the pressure of the system. From eq (7), the pressure of the system can be calculated from the stress tensor as $P=-\left(\left\langle\sigma_{x x}\right\rangle_{\mathrm{eq}}+\left\langle\sigma_{y y}\right\rangle_{\mathrm{eq}}+\left\langle\sigma_{z z}\right\rangle_{\mathrm{eq}}\right) / 3$. If there is no interaction, the pressure simply obeys the equation of state for an ideal gas. Thus the pressure is simply given as that of the ideal gas, $P_{\mathrm{id}}=c \rho$ with $c \rho$ being the number density of molecules in the system. We have $c=1 / 2$ and $c=1$ for the interacting dumbbell systems and soft particle systems, respectively. It would be reasonable to subtract this ideal gas pressure $P_{\text {id }}$ from the pressure and define the excess pressure: $P_{\text {ex }}=P-P_{\text {id }}$. If the target system behaves as an ideal gas, we simply have $P_{\mathrm{ex}}=0$. Figure 4 shows the excess pressure of the interacting dumbbell systems and soft particle systems, which are calculated from stress tensors. In Fig. 4, we clearly observe that the excess pressure is non-zero. As the density $\rho$ and/or the interaction strength $\epsilon$ increases, the excess pressure increases.

Louis et al. ${ }^{23)}$ and Lang et al. ${ }^{24)}$ analyzed static statistical properties of the Gaussian soft-core systems. They found (a)

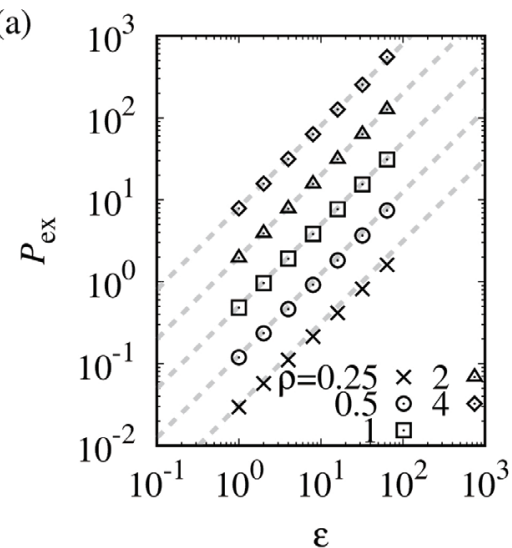

(b)

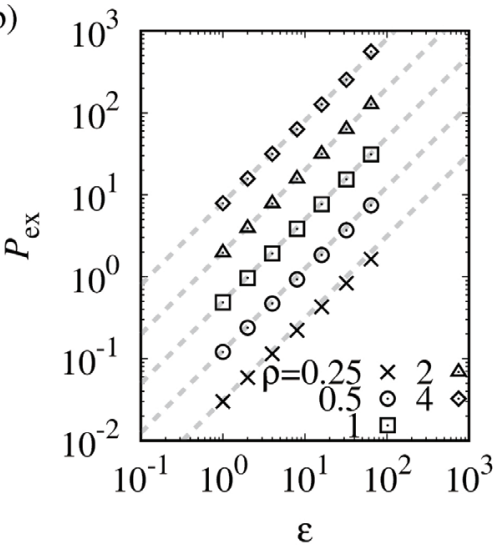

Fig. 4 The excess pressures calculated by the simulations for (a) the interacting dumbbell systems and (b) the soft particle systems, with various densities and interaction parameters. Symbols show the simulation data, and the gray dashed lines represent the theoretical prediction based on the mean-field approximation (eq (13)).

that at the high density limit, the mean-field theory becomes exact. According to their data, the mean-field theory seems to be accurate even if the density is not very high. Lang et al. derived a simple approximate expression for the excess pressure: ${ }^{24)}$

$$
P_{\mathrm{ex}} \approx \frac{1}{2} \epsilon \rho^{2}
$$

(Notice that the definition of the interaction parameter $\epsilon$ in Ref. 24 is different from that in this work.) The prediction by eq (13) is shown in Fig. 4 as gray dashed lines. The agreement between the simulation data and the theoretical prediction is very good even at the low density systems $(\rho=0.25)$. Thus we find that the mean-field approximation works very well for the interacting dumbbell systems and soft particle systems examined in this work.

The static simulation data would be summarized as follows. From the structural data, we found that the structure of the interacting dumbbells approaches to that of the corresponding non-interacting system. However, the pressure data show that the dumbbells are strongly interacting while the 
mean-field approximation works very well. In some aspects, the Gaussian soft-core interaction seems to be canceled and can be ignored, but in some other aspects, it cannot be simply ignored.

\subsection{Linear Viscoelasticity}

From the static data, we cannot judge whether the dynamics of interacting dumbbells can be reasonably approximated by that of non-interacting dumbbells. Judging from the fact that the pressure is not screened, the linear viscoelasticitic properties may also reflect the strong interaction between particles. In the case of ideal non-interacting dumbbells $(\epsilon=0)$, from eqs (6) and (8), we simply have

$$
G(t)=G_{\mathrm{id}}(t)=\frac{\rho}{2} e^{-4 t}
$$

From the static structure data, we expect that the ideal relaxation modulus may be reproduced if the interaction is screened. Thus, by comparing the relaxation moduli of interacting dumbbells with the ideal relaxation modulus, we will be able to judge the screening from the view point of dynamics. (If the interaction in an interacting dumbbell system is actually screened, we expect that $G(t)$ of that system simply reduces to the ideal one, as eq (14).) It would be convenient to use the reduced relaxation modulus $G(t) / \rho$. We show the reduced relaxation moduli of the interacting dumbbell systems with various densities and interaction parameters in Fig. 5. (Note that the curves for $\epsilon=0$ correspond to the relaxation modulus of the ideal system, $G_{\mathrm{id}}(t) / \rho=e^{-4 t} / 2$.) In the exceptional case $\rho=4$ and $\epsilon=64$ (data not shown), the relaxation modulus seems to have very long relaxation time. This might be due to the glassy dynamics, ${ }^{21,22)}$ although we do not go into the detail in this work. If the density is relatively high, we observe that the relaxation moduli depend on the interaction parameter weekly and eq (14) works as a reasonable approximation. If the density is not high, however, we observe that the reduced shear relaxation modulus clearly depends on the interaction parameter. For example, in Fig. 5(a), the relaxation modulus for the case of $\epsilon=64$ is much different from eq (14). The longest relaxation time of the system with $\rho=0.25$ and $\epsilon=64$ is slightly longer than the ideal one. Also, the relaxation modulus has broader relaxation time distribution. The relaxation behavior at the short time region $\left(10^{-2} \lesssim t \lesssim 10^{-1}\right)$ looks rather close to the power-law type relaxation than the single exponential relaxation.

We should recall that the relaxation moduli shown in Fig. 5 contain contributions of the tethering potential and the Gaussian soft-core potential. Therefore, the relaxation behav- (a)

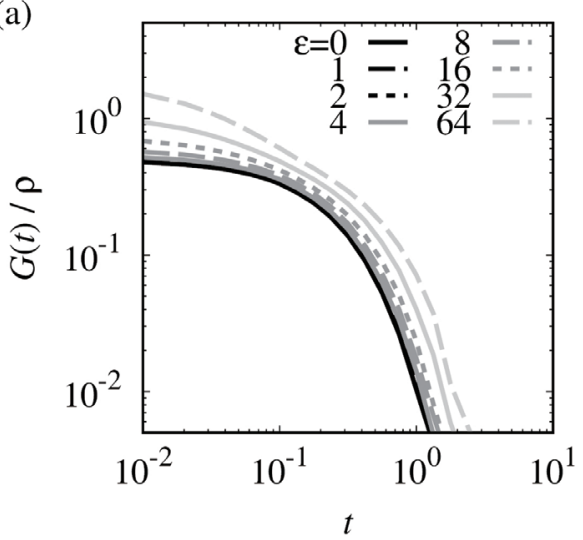

(b)
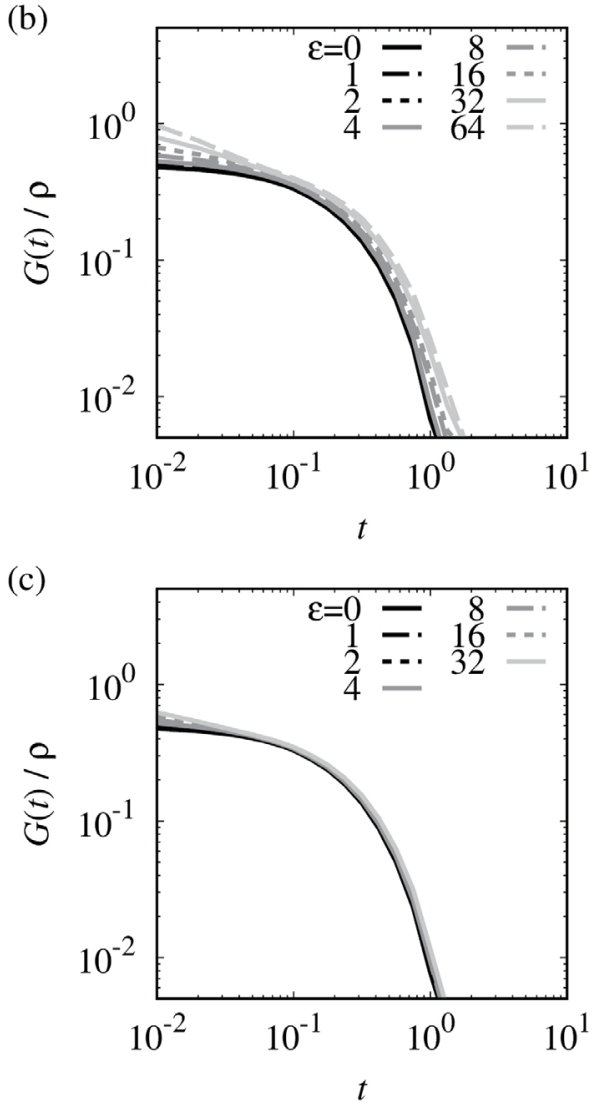

Fig. 5 The shear relaxation moduli for the interacting dumbbell systems. (a) $\rho=0.25$, (b) $\rho=1$, and (c) $\rho=4$. The relaxation modulus for the system with $\rho=4$ and $\epsilon=64$ is much larger than other cases and not shown here. Curves for $\epsilon=0$ (solid black curves) correspond to the relaxation modulus for the ideal system $G_{\mathrm{id}}(t) / \rho$.

ior at the short-time region may be attributed to the Gaussian soft-core interaction. Figure 6 shows the shear relaxation moduli for the soft particle systems. The densities and interaction parameters are the same as those in Fig. 5. The soft particle systems relax around $t \approx 10^{-1}$. If the density is high, the reduced relaxation moduli of soft particle systems are small. Thus the contribution of the Gaussian soft-core interaction will be negligibly small in the high-density interacting dumbbell systems. If the density is low, the reduced relaxation moduli of soft particle systems are rather large. Thus we expect that the relaxation modes due to Gaussian soft-core interaction can be 

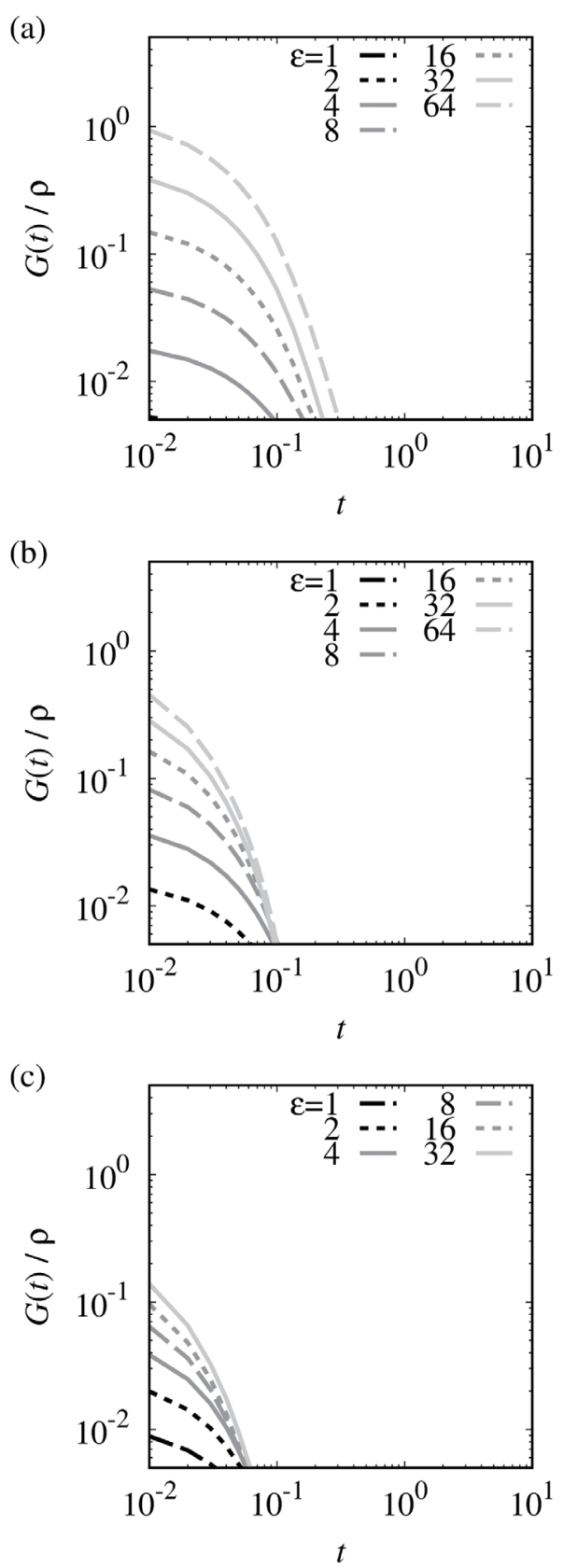

Fig. 6 The shear relaxation moduli for the soft particle systems. (a) $\rho=0.25$, (b) $\rho=1$, and (c) $\rho=4$. The data for $\epsilon=0$ are not shown, since they are trivially zero. Also, the relaxation modulus for the system with $\rho=4$ and $\epsilon=64$ is not shown. The relaxation moduli for relatively small $\epsilon$ may not be observed in the presented range. Simulation parameters are the same as the interacting dumbbell systems shown in Fig. 5.

observed at the short-time region in the interacting dumbbell model, if the density is low. The short-time relaxation modes can be simply attributed to the Gaussian soft-core interaction.

Even if the short-time relaxation modes are attributed to the Gaussian soft-core interaction, the relaxation modes of the tethering potential in the interacting dumbbell systems are generally different from the ideal form (eq (14)). If the bond relaxation mode (the relaxation mode of the tethering potential) is not affected by the Gaussian soft-core interaction, we expect that the relaxation modulus of an interacting dumbbell system can be reconstructed as $G_{\text {dumbbells }}(t)=G_{\text {particles }}(t)+G_{\text {id }}(t)$ with $G_{\text {particles }}(t)$ being the relaxation modulus of the corresponding soft particle system. This reconstruction does not work if the relaxation mode of the tethering interaction is affected by the Gaussian soft-core interaction. If we assume that the effect of the Gaussian soft-core interaction to this relaxation mode can be expressed as the modulation of the relaxation intensity and the relaxation time, we may employ the following empirical form for the relaxation modulus:

$$
\begin{aligned}
G_{\text {dumbbells }}(t) & =G_{\text {particles }}(t)+G_{\text {single }}(t), \\
G_{\text {single }}(t) & =\frac{\rho}{2} \psi_{\text {eff }} e^{-t / \tau_{\text {eff }}} .
\end{aligned}
$$

Here, $G_{\text {single }}(t)$ is the single-mode Maxwell relaxation, and $\psi_{\text {eff }}$ and $\tau_{\text {eff }}$ represent the effective interaction strength and the effective relaxation time, respectively. (In the ideal case where the Gaussian soft-core interaction is absence, we have $\psi_{\text {eff }}=1$ and $\tau_{\text {eff }}=1 / 4$ from $G_{\text {single }}(t)=G_{\text {id }}(t)$.) These parameters should be determined so that the reconstructed relaxation modulus by eq (15) agrees with the simulation data for the interacting dumbbell systems. We show the relaxation moduli by these reconstruction methods together with the simulation data for some interacting dumbbell systems in Fig. 7. The effective relaxation strengths and relaxation times are set as $\left(\psi_{\mathrm{eff}}, \tau_{\mathrm{eff}}\right)=(1.1,0.50)$ for $(\rho, \epsilon)=(0.25,64)$, and $\left(\psi_{\text {eff }}, \tau_{\text {eff }}\right)=(1.0,0.35)$ for $(\rho, \epsilon)=(1,64)$. We observe that the reconstruction with the ideal relaxation modulus does not work while the reconstruction by eq (15) works almost perfectly. From this result, we conclude that the short-time relaxation modes are caused by the Gaussian soft-core interaction, and the long-time bond relaxation mode can be well described by a single-mode Maxwell model. The effective relaxation time becomes slightly longer than that for the ideal system.

\section{DISCUSSIONS}

Although the interaction between dumbbells seem to have rather weak contribution to the shear relaxation modulus from the simulation data (Figs. 5 and 7), we should recall that the dumbbells are strongly interacting each other. This is clear from the pressure data (Fig. 4). The simulation data for the excess pressure simply mean that an interacting dumbbell system (or a soft particle system) can never be treated as an ideal gas. This is not surprising, because we know that polymer chains behave as ideal Gaussian chains in a polymer melt, and at the same time, a polymer melt is almost incompressible. 


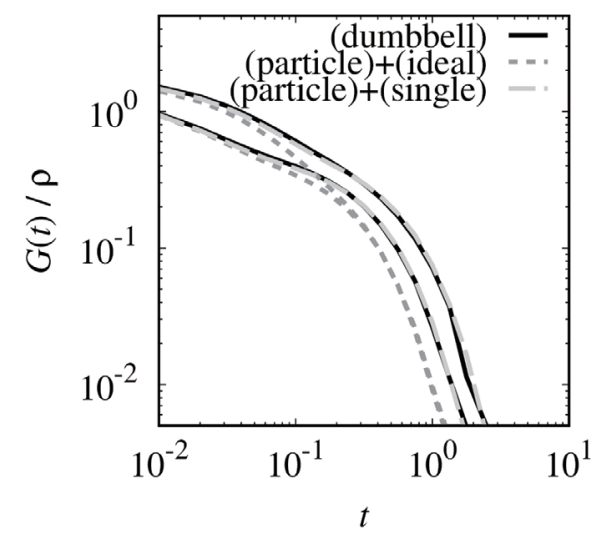

Fig. 7 The shear relaxation moduli for the soft dumbbell systems and the reconstructed shear relaxation moduli. The density and interaction parameter are set as $\rho=0.25$ and 1 , and $\epsilon=64$, respectively. The solid black curves represent the simulation data (taken from Fig. 5). The dark gray dotted curves and light gray dashed curves represent the reconstruction results based on the relaxation moduli for the soft particle systems. The dark gray dotted curves and light gray dashed curves utilize the ideal relaxation modulus (eq (14)) and the singlemode Maxwell model with empirical parameters (eq(16)), respectively.

From eq (13), the compressibility of the system is calculated as

$$
\kappa=\frac{1}{\rho(\partial P / \partial \rho)} \approx \frac{1}{c \rho+\epsilon \rho^{2}}
$$

As the density and/or the interaction parameter increases, the compressibility decreases. In most of polymer melts the interaction is sufficiently strong and the compressibility is sufficiently low. Therefore, we consider that the interacting dumbbell model will be utilized as a simple model for polymer melts, when the density and/or the interaction parameter is high.

As we observed, the shear relaxation moduli of the interacting dumbbells at high density are close to those of noninteracting dumbbells (Fig. 5(c)). A single dumbbell in the system behaves in almost the same way as a non-interacting ideal dumbbell. The situation will not be changed even if we replace interacting dumbbells by interacting Rouse chains. A single Rouse chain in such a system will behave as a noninteracting ideal chain, if the density is sufficiently high. This empirically supports the naive picture of the screening and the application of the Rouse model to unentangled polymer melts.

However, the consideration above implies that the validity of the screening and the recovery of the ideal Rouse dynamics depend on the scale. Here we consider the coarse-graining of a simple Rouse type chain to estimate the scale-dependence of the screening effect. We model a single polymer chain by connecting $N_{0}$ segments by harmonic springs. We assume that segments are already coarse-grained and the interaction between segments can be approximately expressed by a Gaussian soft-core potential. We express the segment size as $b_{0}$ and the segment density as $\rho_{0}$. We define a coarse-grained segment which consists of $\alpha$ segments. Then the polymer chain is reinterpreted as a chain in which $N_{1}$ segments are connected by harmonic springs. The coarse-grained number of segments and segment size are $N_{1}=\alpha^{-1} N_{0}$ and $b_{1}=\alpha^{1 / 2} b_{0}$. The segment density is changed as $\rho_{1}=\alpha^{-1} \rho_{0}$. From the viewpoint of the interacting dumbbell and soft particle models, we should use the dimensionless segment density. For the models before and after the coarse-graining, we have $\rho_{0} b_{0}^{3}$ and $\rho_{1} b_{1}^{3}=\alpha^{1 / 2}\left(\rho_{0} b_{0}^{3}\right)$. Therefore, by the coarse-graining, the dimensionless segment density increases by the factor $\alpha^{1 / 2}$. The interaction parameter is also changed by this coarse-graining. We express the interaction parameter before and after the coarse-graining as $\epsilon_{0}$ and $\epsilon_{1}$. Under the mean-field approximation, the interaction energy per unit volume should be invariant under the coarse-graining: $\epsilon_{0} \rho_{0}^{2} / 2=\epsilon_{1} \rho_{1}^{2} / 2$. Thus we have $\epsilon_{1}=\alpha^{2} \epsilon_{0}$. We should use the dimensionless unit to discuss the effective interaction strength. The dimensionless interaction parameters are roughly estimated to be $\epsilon_{0} / k_{B} T b_{0}^{3}$ and $\epsilon_{1} / k_{B} T b_{1}^{3}$, and then we have $\epsilon_{1} / k_{B} T b_{1}^{3}=\alpha^{1 / 2}\left(\epsilon_{0} / k_{B} T b_{0}^{3}\right)$. This means that the dimensionless interaction parameter increases by the factor $\alpha^{1 / 2}$ by the coarse-graining.

From the estimates shown above, both the dimensionless segment density and the dimensionless interaction parameter change as the coarse-graining is performed. If the coarse-graining is performed further, the dimensionless segment density increases further, and the mean-filed description for the Gaussian soft-core interaction becomes more accurate. Although the interaction parameter also increases with the coarse-graining, the relaxation modulus is not sensitive to the interaction parameter (as far as the dimer-like clusters are not formed). As a result, at a high coarse-graining level, the screening effect is safely justified. The lower order Rouse modes corresponds to such highly coarse-grained dynamics, and such Rouse modes would behave in almost the same way as in ideal non-interacting systems. This result would also justify some coarse-grained multi-chain models which are based on the non-interacting ideal chain statistics. ${ }^{25,26)}$

On the other hand, at relatively small scales where the coarse-graining level is low, the screening is not fully justified. Thus the higher order Rouse modes may deviate from the ideal behavior. We should recall that we observed the relaxation modes by the Gaussian soft-core interaction at the short-time scale region. The Rouse relaxation modes will be mixed-up with the Gaussian soft-core interaction modes. For the case of the dumbbell model, we observed that the 
relaxation modulus apparently looks power-law like if the interaction is not screened. If we naively consider the situation is similar for the higher order Rouse modes, we will observe the sum of power-law like relaxations at the short-time region. The sum of Rouse relaxation modes itself is already a power-law like relaxation (with the power-law exponent 1/2). Then, even if the Gaussian soft-core interaction modulates a Rouse relaxation mode from a single exponential form to a power-law form, the resulting relaxation modulus may just look like a power-law relaxation. Therefore we expect that we may still observe the Rouse-like power-law relaxation at the short time region, even if the screening does not work and the contribution of the Gaussian soft-core interaction remains. Only from the Rouse-like functional form of the relaxation modulus, we may not be able to judge whether the interaction between the segments is really screened or not. We may need to carefully analyze several dynamical quantities to judge the screening effect experimentally. Conversely, when we study the linear viscoelastic properties, the screening effect may be naively assumed (because the results will not be sensitive to the screening effect).

Another example where the screening may not be fully justified is a polymer solution (at the theta point). In a polymer solution, the effective density of polymer chains can be rather low. Then the screening effect may be insufficient and the bond length distribution and the relaxation modulus may deviate from the ideal ones. Then, even for lower order relaxation modes, we may observe the deviation from the ideal behavior. However, we should recall that there is the hydrodynamic interaction in a polymer solution. The hydrodynamic interaction may not be screened, when the interaction potential is not fully screened. Then the relaxation modulus will be affected by both the hydrodynamic interaction and the interaction potential. The hydrodynamic interaction strongly affects the relaxation modulus (as predicted by the Zimm model), and thus is primarily important. The effect of the interaction potential may be secondary and may not be clearly observed even if it exists.

Before we end this section, we should recall that we have examined the screening effect only in equilibrium. This means that we cannot discuss the nonlinear rheological behavior based on our simulation results. Hess considered an interacting dumbbell system and proposed a constitutive equation model. ${ }^{27)}$ Hess constructed an effective dynamics model for a single dumbbell under the mean-filed approximation. The effects of the interaction between dumbbells is expressed as the effective tethering interaction potential with the effective interaction matrix. This effective interaction matrix reflects the average conformational change, and thus we observe nonlinear rheological behavior such as the shear thinning behavior. From the view point of nonequilibrium statistical mechanics, it would be reasonable to introduce the mean-field effective mobility ${ }^{8)}$ instead of the effective tethering potential. In this picture, the mobility (or the friction coefficient) is modeled as an anistropic tensorial quantity which reflects the average conformational change. In both cases, we observe that the rheological behavior is largely changed under fast shear flows. This is consistent with the experimental fact that unentangled polymer melts exhibit shear thinning behavior which cannot be described by the Rouse model. We will need to perform additional simulations under nonequilibrium conditions, to study how the screening effect works in nonequilibrium states and how we can model the nonequilibrium dynamics. It is beyond the scope of the current work and will be an interesting future work.

\section{CONCLUSIONS}

We have performed simulations for interacting soft dumbbell systems and soft particle systems, to study the screening effect. From the static structural data (the RDFs and the bond length distributions), we found that the screening reasonably works if the density is high. This does not mean that an interacting dumbbell system becomes approximately equivalent to the corresponding non-interacting ideal dumbbell system. The pressure increases as the density increases, and thus an interacting dumbbell system becomes less compressible as the density increases.

The relaxation moduli of the interacting dumbbell exhibit similar screening effect. For high density systems, the relaxation moduli are very close to the relaxation moduli of the non-interacting ideal dumbbell systems. This result means that the screening effect works both for static structures and dynamics of interacting dumbbells. Our simulation results would partly support the usage of the naive screening picture in various coarse-grained dynamics model for polymer melts. If the density is low, we observe the relaxation modulus deviates from the ideal one. However, the short-time relaxation modes observed in low density systems can be attributed to the relaxation modes of the Gaussian soft-core interaction. The bond relaxation mode can be expressed as a single Maxwell relaxation mode, although the relaxation intensity and relaxation time are slightly different from ideal ones. If we assume that the situation is similar in the case of the interacting Rouse chains, we expect that the relaxation modulus of the interacting Rouse model will be indistinguishably similar to the ideal 
Rouse model without interaction between segments.

\section{ACKNOWLEDGMENT}

This work was supported by JST, PRESTO Grant Number JPMJPR1992, Japan, and Grant-in-Aid (KAKENHI) for Transformative Research Areas B JP20H05736.

\section{REFERENCES}

1) Doi M, Edwards SF, The Theory of Polymer Dynamics (1986), Oxford University Press, Oxford.

2) Kawakatsu T, Statistical Physics of Polymers: An Introduction (2004), Springer Verlag, Berlin.

3) de Gennes PG, Scaling Concepts in Polymer Physics (1979), Cornell University Press, Ithaca, New York.

4) Rouse PE, J Chem Phys, 21, 1272 (1953).

5) Likhtman AE, Sukumaran SK, Ramirez J, Macromolecules, 40, 6748 (2007)

6) Hansen JP, McDonald IR, Theory of Simple Liquids 3rd ed, (2006), Elsevier, Amsterdam.

7) Kröger M, Phys Rep, 390, 453 (2004).

8) Uneyama T, Horio K, Watanabe H, Phys Rev E, 83, 061802 (2011).

9) Watanabe H, Matsumiya Y, Nihon Reoroji Gakkaishi (J Soc Rheol Jpn), 45, 185 (2017).

10) Watanabe H, Matsumiya Y, Sato T, Nihon Reoroji Gakkaishi ( $J$ Soc Rheol Jpn), 48, 259 (2020).

11) Uneyama T, Miyaguchi T, Akimoto T, Phys Rev E, 99, 032127 (2019).
12) Miyaguchi $T$, Uneyama $T$, Akimoto $T$, Phys Rev E, 100, 012116 (2019).

13) Uneyama T, Nakai F, Masubuchi Y, Nihon Reoroji Gakkaishi (J Soc Rheol Jpn), 47, 143 (2019).

14) Uneyama T, Nihon Reoroji Gakkaishi (J Soc Rheol Jpn), 37, 81 (2009).

15) Louis AA, Bolhuis PG, Hansen JP, Meijer EJ, Phys Rev Lett, 85, 2522 (2000).

16) Addison CI, Hansen JP, Krakoviack V, Louis AA, Mol Phys, 103, 3045 (2005).

17) Honeycutt RL, Phys Rev A, 45, 600 (1992).

18) Evans DJ, Morris GP, Statistical Mechanics of Nonequilibrium Liquids, 2nd ed, (2008), Cambridge University Press, Cambridge.

19) http://www.polymer-physics.jp/uneyama/en/spreads.html

20) Likhtman AE, in Polymer Science: A Comprehensive Reference, Matyjaszewski K Möeller M eds, vol 1, pp 133-179, (2012), Elsevier, Amsterdam.

21) Miyazaki R, Kawasaki T, Miyazaki K, Phys Rev Lett, 117, 165701 (2016).

22) Miyazaki R, Kawasaki T, Miyazaki K, J Chem Phys, 150, 074503 (2019).

23) Louis AA, Bolhuis PG, Hansen JP, Phys Rev E, 62, 7961 (2000).

24) Lang A, Likos CN, Löwen MWH, J Phys: Cond Mat, 12, 5087 (2000).

25) Uneyama T, Masubuchi Y, J Chem Phys, 137, 154902 (2012).

26) Uneyama T, J Chem Phys, 150, 024901 (2019).

27) Heß W, Rheol Acta, 23, 477 (1984). 